\title{
Effet potentiel des apports d'azote d'origine fluviale sur le phytoplancton marin entre Roscoff et Saint-Brieuc
}

\author{
Joëlle Riou et Philippe Gosse
}

Electricité de France, Dépt. Environnement aquatique et atmosphérique - Chatou

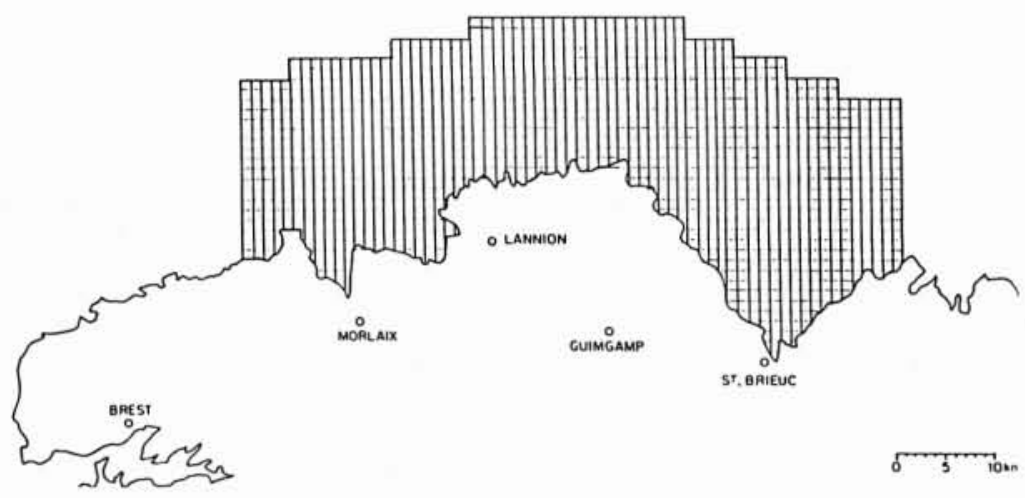

1. Côte Nord Bretonne, emprise du modèle et maillage.

\section{Introduction}

Les conséquences de l'utilisation et des rejets de produits azotés sont très perceptibles sur les eaux superficielles continentales et sur les nappes phréatiques dont les teneurs en nitrates ne cessent de croitre sur le territoire national depuis plusieurs années.

Les départements du Finistère et des Côtes-du-Nord sont très touchés par cette évolution, les eaux souterraines ne respectant pas les normes CEE de potabilité en de nombreux endroits.

On commence à mesurer l'impact sur le milieu marin de ces apports d'azote, notamment dans le panache des grands fleuves (Loire, Seine) où peuvent apparaître des concentrations élevées de phytoplancton, ou algues microscopiques vivant au fil de l'eau. Sur la côte nordbretonne, des proliférations d'Ulves (algues macroscopiques au départ fixées sur le fond, communément appelées
"Laitues de mer") apparaissent dans la zone de rejet de certains fleuves à débit modéré comme le Gouessant et le Yar (Menesguen et SAlomon, 1988).

Ces manifestations particulièrement spectaculaires touchent le très proche littoral nord-breton et beaucoup s'interrogent - ici comme sur d'autres mers - sur l'impact de ces apports terrigènes d'azote à une distance de plusieurs kilomètres au large; sur la côte nord-bretonne, c'est un domaine où cohabitent deux types de végétaux dont la production annuelle est de même ordre de grandeur (ChAsse, 1983) : le phytoplancton et les macrophytes ou végétaux fixés macroscopiques, essentiellement composés de laminaires à croissance plus faible que les Ulves.

L'objet de cet article est de répondre à cette question en évaluant l'impact sur le phytoplancton des apports azotés des fleuves nord-bretons sur une bande côtière de largeur 30 kilomètres entre Roscoff et St-Brieuc (voir fig. l).

\section{Potential effect of nitrogen from rivers on the marine phytoplankton between Roscoff and Saint-Brieuc}

A first version of a digital model of a coastal ecosystem developped in North Brittany between Roscoff and Saint-Brieuc is described. Designed for impact studies and taking into account a meterological time scale of over 20 years, this model simulates the daily changes in water temperature, salinity, phytoplanktonic biomass and nitrogen, with spacing of $2 \mathrm{~km}$. After indicating the principal adjustment phases of the model and actions planned to improve its descriptive quality, an application of the latter is given showing possible consequences of nitrogen from the coastal rivers, in an agricultural region with heavy use of fertilizers, on the development of marine phytoplankton. 


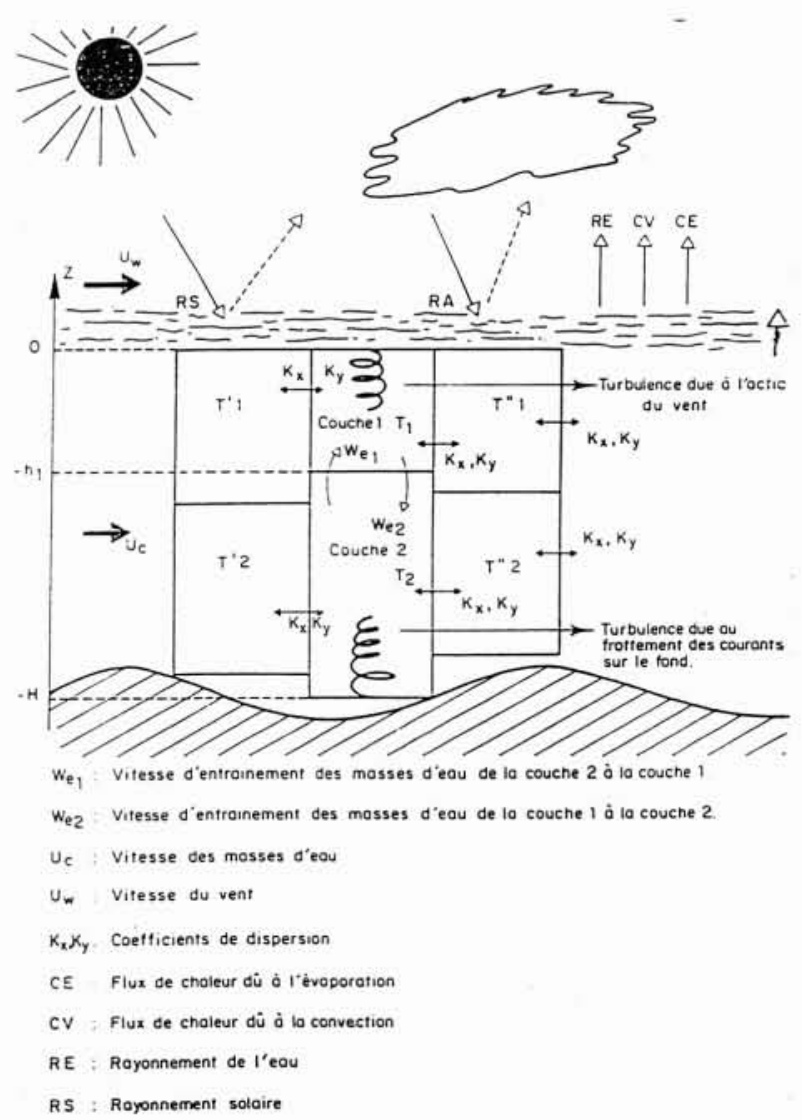

2. Schématisation des mécanismes de la partie thermique du modèle.

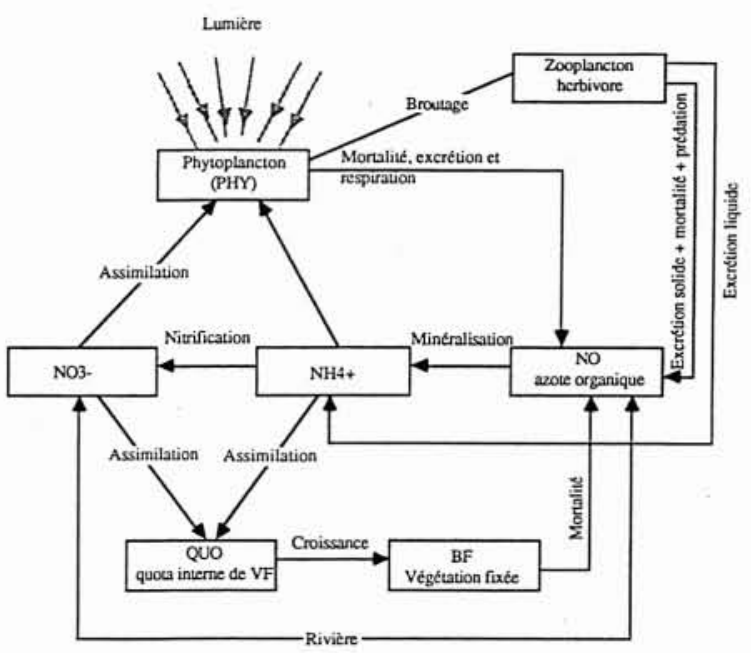

3. Représentation schématique de l'écosystème phytoplanctonique du modèle "côtier".

On utilise pour cela un modèle numérique déterministe qui, couplé avec un modèle thermique, simule sur plusieurs cycles annuels - avec un pas de temps de une heure les variables biomasse phytoplanctonique, biomasse zooplanctonique herbibore, biomasse des laminaires et azote sous plusieurs formes.
Après une présentation des caractéristiques du modèle $(\S 2)$, on examine sa qualité descriptive ( $\$ 3$ ), puis on visualise l'impact des rejets fluviaux en simulant un arrêt total des apports terrigènes d'azote $(\S 4)$.

\section{Description du modèle}

\subsection{Rappel du modèle}

Le modèle côtier mis au point reprend la conception du modèle d'écosystème phytoplanctonique (dit modèle global) développé sur toute la Manche par AgoumI (1985) : il est bidimensionnel sur le plan horizontal avec deux couches homogènes d'épaisseurs variables sur la verticale ; il simule, sur des cycles pluriannuels, l'ensemble des variables du modèle global en conservant la formulation de leurs équations de bilan et les valeurs des paramètres d'ajustement, à l'exception du coefficient de turbidité qui est déduit directement des mesures de la campagne SMART (voir §3); d'autre part, il prend en compte la courantologie résiduelle due au vent et à la marée et l'effet dispersif de la marée.

Cependant des différences apparaissent :

- la description spatiale et temporelle a été affinée (pas d'espace $2 \mathrm{~km} \times 2 \mathrm{~km}$, et pas de temps de 1 heure), en utilisant des données bathymétriques et courantologiques fournies par le Laboratoire National d'Hydraulique (Regimbaud, 1987). Cette nouvelle échelle spatiale nécessite un recalage des coefficients de diffusion, décrivant l'effet dispersif des marées;

- le modèle côtier ne génère pas lui-même, contrairement au modèle global, ses conditions aux limites en mer ouverte; celles-ci sont issues des résultats de simulation du modèle global, dans lequel est modifié, par rapport à la version présentée par Agoumi (1985): le stock d'azote total pris égal à $14 \mu \mathrm{atgN} / 1$ au début de la simulation, le rapport intracellulaire $\mathrm{C} / \mathrm{Chla}$ pris égal à 2,7 dans le phytoplancton et la constante de demi-saturation XKN (traduisant l'effet de l'azote sur la croissance phytoplanctonique) prise égale à $0,01 \mathrm{mgN} / 1$; ces nouvelles conditions, conservées dans le modèle côtier, assurent une assez bonne représentation des conditions aux limites de ce dernier modèle ; - les rejets côtiers et le compartiment végétal benthique sont pris en compte, cette démarche étant indispensable pour une bonne représentation de la biomasse phytoplanctonique à l'échelle considérée (voir § 4 , et fig. 12, 13, 14 et 15).

* Partie thermique du modèle: Le principe de ce sousmodèle (fig. 2) est de calculer les températures de surface et de fond d'un milieu aquatique, soumis à des échanges d'énergie à l'interface eau-atmosphère (ces échanges sont calculés à partir des observations atmosphériques trihoraires de la station météorologique de la Hague, représentative de la Manche), et à des turbulences générées par le vent en surface et les courants de marée au fond; celles-ci modifient l'épaisseur des deux couches, en entraînant les masses d'eau d'une couche à l'autre par l'intermédiaire de vitesses (dites vitesses d'entraînement) calcu- 
lées par intégration des équations de l'énergie cinétique turbulente sur chaque couche.

Partie biologique du modèle: Les variables prises en compte sont: d'une part la biomasse phytoplanctonique prise globalement sans distinction d'espèce (PHY), le zooplancton herbivore (ZOO), l'azote organique détritique (NO), lazote ammoniacal $\left(\mathrm{NH}_{4}\right)$, l'azote nitrique $\left(\mathrm{NO}_{3}\right)$, ensemble de variables qui subissent les échanges horizontaux et verticaux comme la variable température; d'autre part la biomasse végétale benthique (BF) et le quota intracellulaire en azote de cette biomasse (QUO), qui n'échangent de l'azote qu'avec la couche de fond. Les différents processus considérés sont représentés sur la figure 3 .

Le système d'équations différentielles régissant le modèle est rappelé dans le tableau $I$ (page suiv.) ainsi que les valeurs des paramètres et fonctions considérées ( $t a b l .2 \mathrm{et}$ $3)$. Pour plus de détails sur cette modélisation, nous renvoyons le lecteur aux écrits d'Agoumi (1985), et AgOUMI, Gosse, Khalanski (1985) ; nous ne développerons ici que la partie originale du modèle côtier, décrite plus amplement dans la thèse de Riou (à paraître).

\subsection{Les apports fluviaux}

Le SRAE a estimé à 4600 tonnes de nitrates les rejets déversés en 1981 par les rivières sur la bande côtière étudiée (fig. 4) (Assolre, 1985). A partir des données de débits des principales rivières du littoral (avec d'Est en Ouest, le Gouessant, le Gouet, le Leff, le Trieux, le Jaudy, le Guindy, Le Léguer, le Yar, le Tromorgant, le Queffleuth, le Jarlot et la Penzé), et des mesures des concentrations en nitrates relevées sur chacun de ces cours d'eau par différents organismes publics régionaux (DDE, DDA, SRAE, Agence de Bassin), nous avons reconstitué les concentrations journalières de nitrates de ces rivières à partir de la formule du type : $\left[\mathrm{NO}_{3}\right] \mathrm{mgN} / \mathrm{l}=a_{i} Q_{i}(t) \mathrm{m}^{3} \cdot \mathrm{s}^{-1}, a_{i}$ étant spécifique à chacune des rivières et $Q_{i}$ le débit de la rivière concernée (tabl.4). Cette formulation permet de retrouver les estimations de flux annuels faites, pour l'année 1981, par le SRAE.

\subsection{La végétation fixée}

Si l'idée fondamentale qui a animé ce travail consiste à décrire de façon simple, tout en étant suffisamment réaliste, la dynamique de développement du phytoplancton pris globalement (c'est-à-dire sans distinction d'espèce, ce qui explique le choix unique de l'azote comme élément nutritif limitant), on ne peut pas dans la bande côtière (voir résultats du modèle au paragraphe suivant), négliger les effets de la végétation fixée, dont CHASSE (1983) estime qu'elle représente au moins $50 \%$ de la production primaire des fonds inférieurs à 50 mètres. Près de $80 \%$ de cette production étant due, dans les eaux bretonnes, aux grands champs de laminaires, le compartiment végétal benthique simulé est basé sur les données des caractéristiques principales de ces algues.

A partir des cartes de superficies rocheuses établies par KERAMBRUN (1984) pour des fonds inférieurs à 12 mètres (fig. 5) et des données de CHASSE et al. (1985) concernant

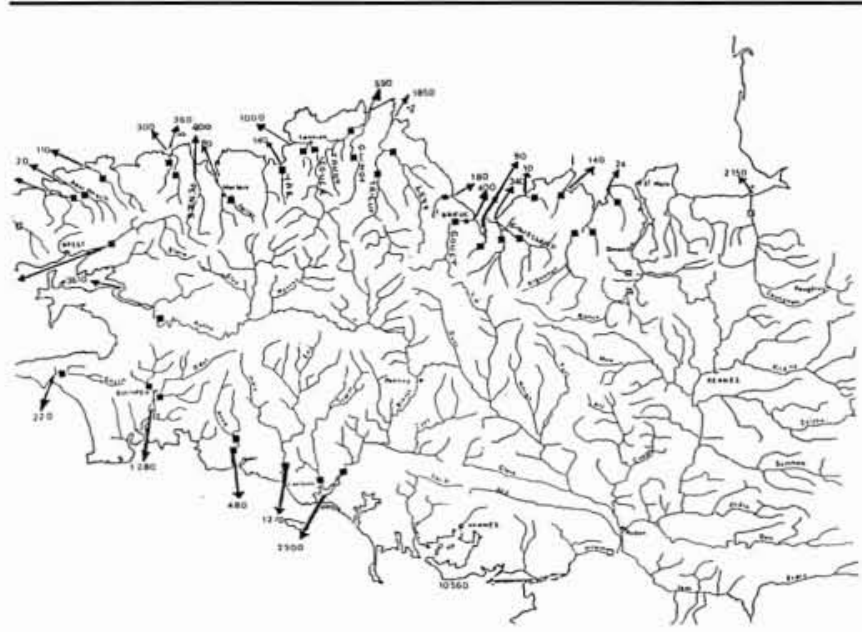

4. Apports annuels d'azote nitrique en tonnes d'azote aux points de contrôle (-) année 1981 (SRAE 1985).

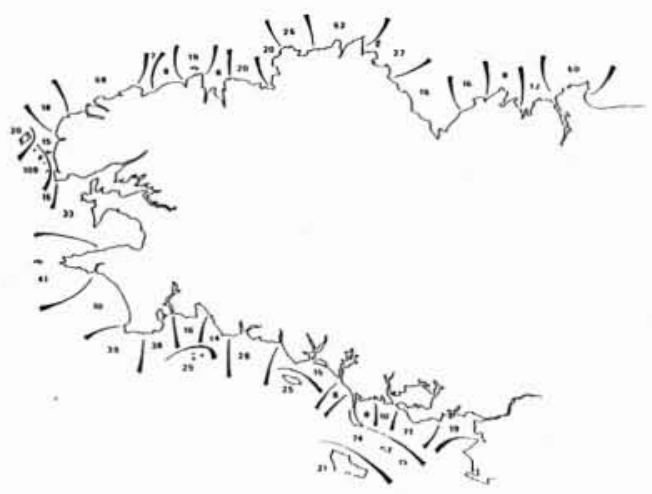

5. Evaluation par secteur des surfaces du champ d'algues de Bretagne en $\mathrm{km}^{2}$ ( 4 départements). Surfaces rocheuses entre 0 et $12 \mathrm{~m}$ y compris l'estran (d'après KerAMBrun, 1984).

les fonds de 0 à 50 mètres, nous avons pu définir, pour chaque maille du domaine, la biomasse algale au $1^{\text {er }}$ janvier (début de la simulation), celle-ci étant supposée nulle pour les fonds supérieurs à 50 mètres.

Les conditions initiales étant définies, le sous-modèle benthique a été construit sur la base des observations réalisées par KERAMBRUN sur les champs de Laminaires en Bretagne. On suppose que :

- en moyenne sur le domaine d'étude, la croissance annuelle est équilibrée par les pertes subies par mortalité ou arrachement, ce qui implique un retour en fin de cycle annuel à une densité du champ algal comparable à celle de départ :

- la croissance ne s'effectue que pendant les six premiers mois de l'année, avec environ un doublement de la biomasse pour les fonds compris entre 0 et 12 mètres;

- la biomasse fixée a la capacité, en période hivernale, d'assimiler l'azote minéral dissous, et de puiser dans ses réserves lorsque l'eau s'est appauvrie au printemps. Ce mécanisme est simulé par l'introduction de la variable Quota interne en azote, dont la plage de variation $(Q \min$ et $Q \max )$ est donnée par Kerambrun.

Pour conserver au cours de la simulation la répartition 


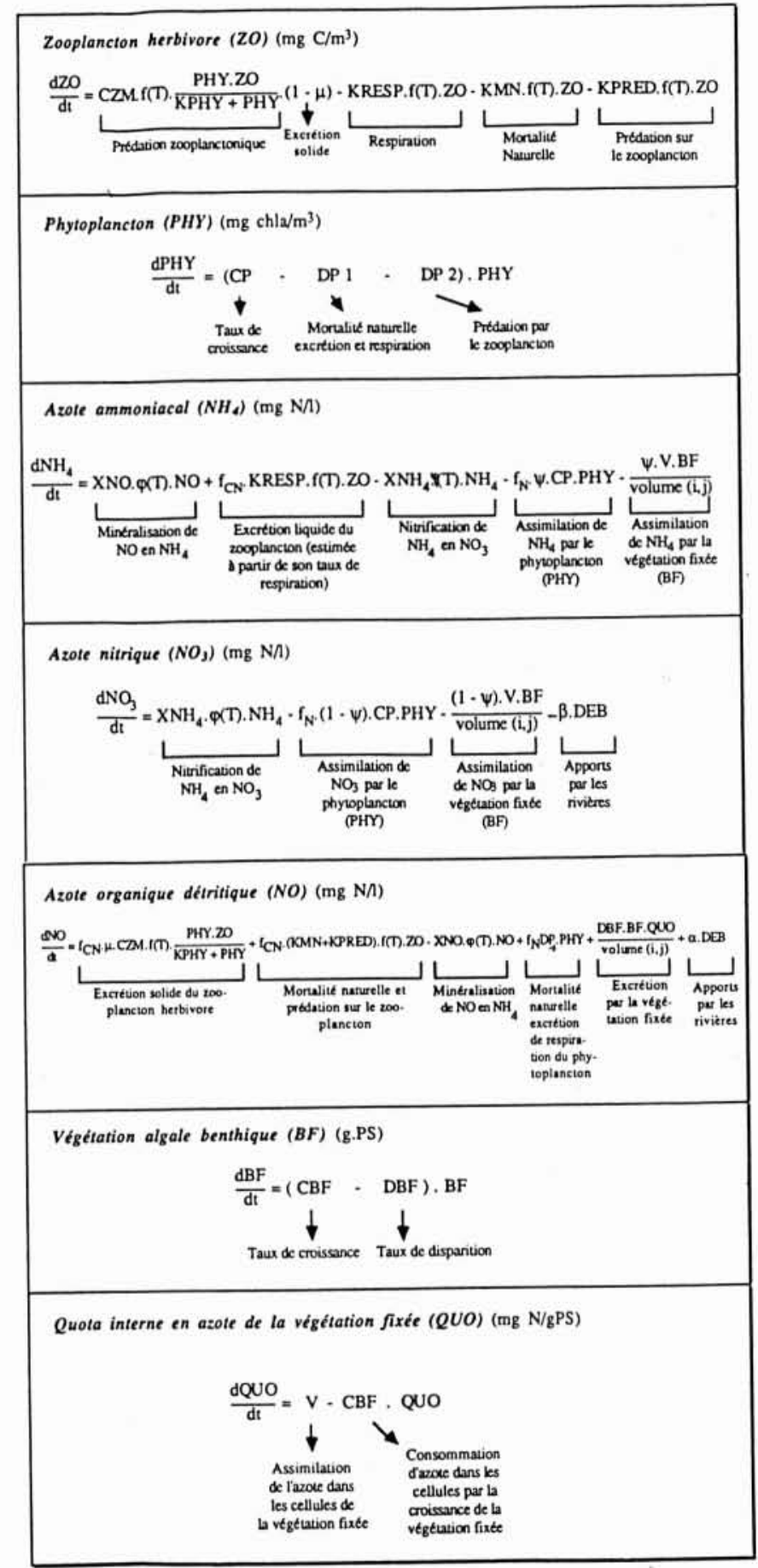

\begin{tabular}{|c|c|c|c|}
\hline Foncrioss & Sicsultation & 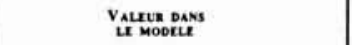 & Rerenences \\
\hline CP & $\begin{array}{l}\text { Teas de croisunce du } \\
\text { phylopianction }\end{array}$ & 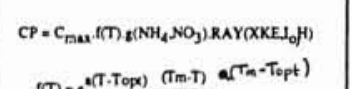 & \\
\hline (T) & Elter de is temperature nur CP & 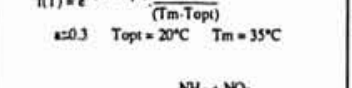 & Loi de Lessite el Keams \\
\hline $8\left(\mathrm{NH}_{4}, \mathrm{NO}_{3}\right)$ & Elles des nutrimenu nur CP & 1) $=\frac{\overline{\mathrm{XON}}+\mathrm{NH}_{4}+\mathrm{NO}_{3}}{4}$ & Kremet et Nixon (1978) \\
\hline Rey $\left(X X D_{0} \cdot H\right)$ & 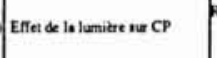 & HXXE & Steetle \\
\hline$X O E$ & 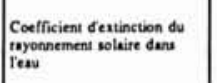 & $\mathrm{XKE}=\mathrm{XKE} E_{0}+0.0088 \mathrm{PHY}+0.05 \mathrm{PHY} \mathrm{Y}^{23}$ & Riley (1979) \\
\hline (T) & 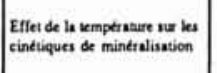 & $\cdot(\pi)=(1.05)^{(\pi-20)}$ & Cosse (1982) \\
\hline DP1 & 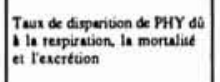 & $D P 1=m(1.06)(-20)$ & \\
\hline $\mathrm{DP} 2$ & Taud de divpention de PHY dü & $D P Z=C Z M \cdot I(T) \frac{D D}{K P H Y, P H Y} \cdot I Z P+P P H Y>0 S$ & $\mathrm{me}_{\mathrm{C}} \mathrm{CNim}^{3}$ \\
\hline & 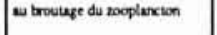 & siPHY $<0$ of & me $\mathrm{CAll}_{2} \mathrm{~m}^{3}$ \\
\hline $\mathrm{CBF}$ & 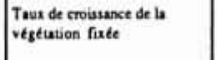 & $\mathrm{CBF}=\mathrm{CBF}_{\operatorname{mex}} \cdot \mathrm{PO} \cdot \mathrm{B}(\mathrm{T})$ & \\
\hline p(t) & Eiftet \& is lunizes sur CBF & Fonction nevile etimes $10.05 \mathrm{~W} / \mathrm{m}$ & Drew ea a (1989) \\
\hline (n) & Ellet de it temptrasure aur CBH & $r(T)=\frac{1}{\left(1+19 \exp ^{-054(\pi-1)}\right)}$ & Menesguen (isn) \\
\hline $\mathrm{v}$ & Vilese destimilation & $\left.\mathrm{v}=\mathrm{v}_{\max } \cdot \mathrm{HNO}_{3} \mathrm{~N} \mathrm{NH}_{4}\right)(\mathrm{O})$ & \\
\hline "(NO3NA4) & Ellet des nustiments sut V & $\mathrm{h}\left(\mathrm{NO}_{3} \mathrm{NH}_{4}\right)=\frac{\mathrm{NH}_{4}+\mathrm{NO}_{3}}{\mathrm{~K}_{3}+\mathrm{NO}_{3}+\mathrm{NH}_{4}}$ & \\
\hline$\varphi(0)$ & EStet du quou sur V & $\varphi(Q)=\frac{Q \cdot Q_{\min }}{Q_{\operatorname{mex}} \cdot Q_{\min }}$ & \\
\hline
\end{tabular}

TABlEAU 4 : COEFFICIENTS CARACTÉRISTIQUES DE CHAQUE RIVIERE DANS LA FORMULATION DES REJETS AZOTES

\begin{tabular}{|c|c|c|c|c|c|c|c|c|c|c|c|c|}
\hline Rivitete & Gouesan & Govet: & 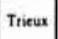 & Leff & Iowaly & Guindy & logeret & $Y_{*}$ & Jelor| & Tromorgant & Queflewert & Penst \\
\hline$a_{i}$ & 1,72 & 2.6 & 2.76 & 1.37 & 1,11 & 3.6 & 0.6 & 3,87 & 0.2 & 0.95 & 0,3 & 0.73 \\
\hline
\end{tabular}


de la végétation fixée sur un gradient côte-large et obtenir un démarrage de sa croissance à partir de février-mars, on considère un taux de croissance de la végétation fixée dépendant de la lumière et de la température.

On remarquera que les végétaux à très forte vitesse de croissance, de type Ulves, qui apparaissent très près de l'embouchure de la rivière du Gouessant ne sont pas simulés dans le modèle. Cependant ils sont pris en compte en supposant que leur action se traduit par une rétention des nitrates déversés par le Gouessant, ce piégeage étant estimé à partir des travaux de Menesguen et SAlomon (1988).

\section{Qualité descriptive du modèle}

Une campagne de mesures spécifiques a été réalisée sur le littoral Nord-Breton entre juin 1987 et juin 1988 (campagne SMART) (fig. 6) (GosSE, Riou, VIDEAU, à paraître). Elle a permis d'effectuer un ajustement du modèle en trois radiales côte-large du domaine d'étude, ce nombre relativement élevé de points de calage allant dans le sens d'une meilleure fiabilité du modèle. Les données relatives à la température étant abondantes sur la zone étudiée, les paramètres de diffusion, décrivant l'effet dispersif des marées, ont été ajustés par l'intermédiaire de la variable température en utilisant deux sources de données: les mesures réalisées entre 1960 et $1970^{-}$par l'Institut Scientifique et Technique des Pêches Maritimes (I.S.T.P.M.) en trois stations en baie de Morlaix (fig. 6) et les résultats de la campagne SMART (fig. 7). Le sous-modèle thermique a ensuite été vérifié sur les suivis journaliers réalisés en 1985 à l'estacade de Roscoff par Sournia et al. (1987): la figure 8 montre ainsi la bonne réponse du modèle en des points extrêmement côtiers. La précision des températures journalières données par le modèle est meilleure que le degré celsius. Une illustration d'application pluri-annuelle du modèle est donnée sur la figure 9 où sont présentées des statistiques de dépassement du seuil $17^{\circ} \mathrm{C}$ sur une chronique de 25 ans (Gosse et Riou, 1989).

Le calage des différents paramètres des équations de bilan du quota intracellulaire en azote et de la biomasse végétale benthique a été fait de façon, premièrement à respecter les hypothèses relatives à cette biomasse énoncées au paragraphe précédent, et deuxièmement à reconstituer les profils annuels (obtenus avec la campagne SMART)

6. Points de mesure.

- stations de la campagne SMART 1987
X stations de l'ISTPM 1960-1970

E estacade 1985 .

7. Résultats du modèle thermique en 1987. Comparaison avec les points de mesure de la campagne SMART.

8. Résultats du modèle thermique en 1985. Comparaison avec les points de mesure de l'Estacade.

9. Côte de Bretagne-Nord: nombre de jours par an pendant lesquels la température moyenne de la colonne d'eau est supérieure à $17^{\circ} \mathrm{C}$. Statistiques sur les années 1962 à 1987 issues soit du modèle numérique global de la Manche (mailles de $11 \times 11 \mathrm{~km}^{2}$ en pointillé, avec les chiffres correspondants cerclés et placés dans le coin droit) soit du modèle numérique côtier Bretagne-Nord (mailles de $2 \times 2 \mathrm{~km}^{2}$ avec les chiffres indiqués au centre de la maille).

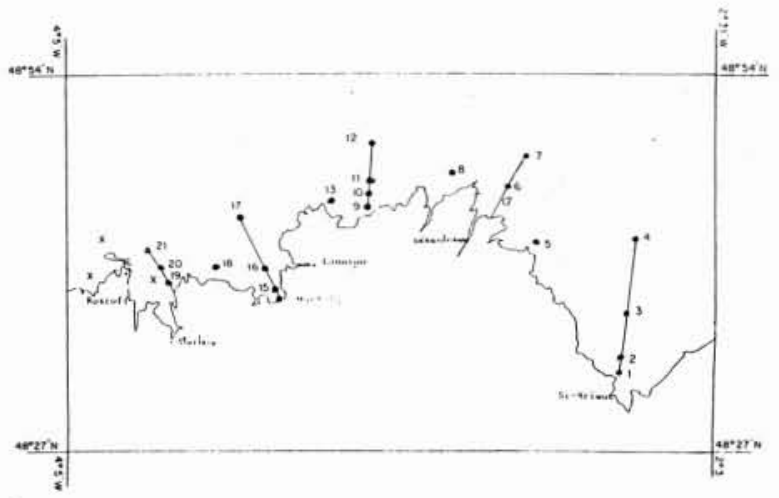

6.
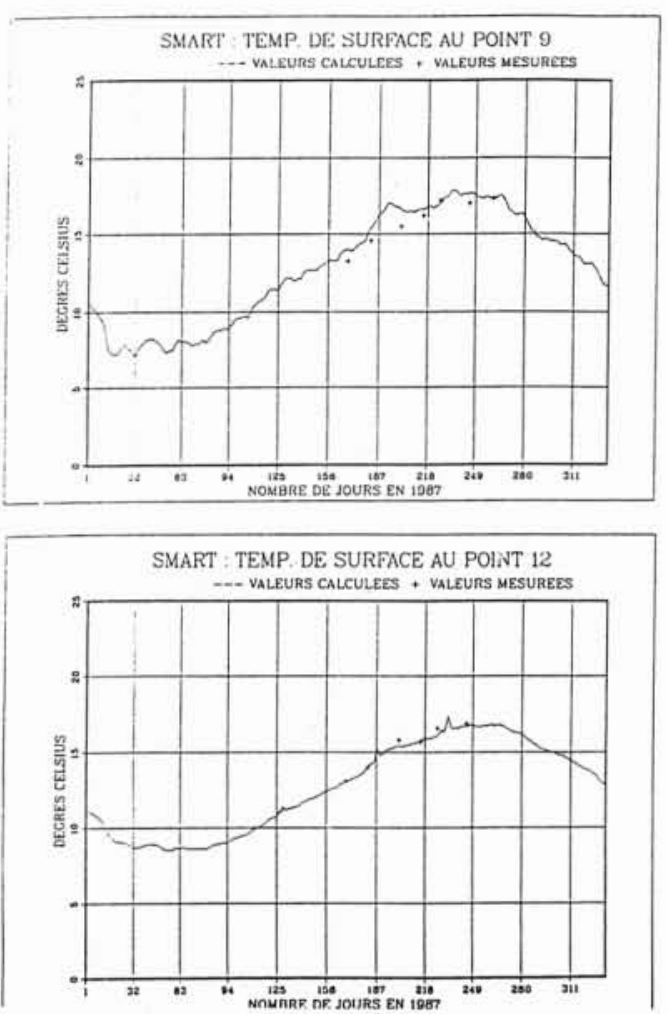

7.

8.

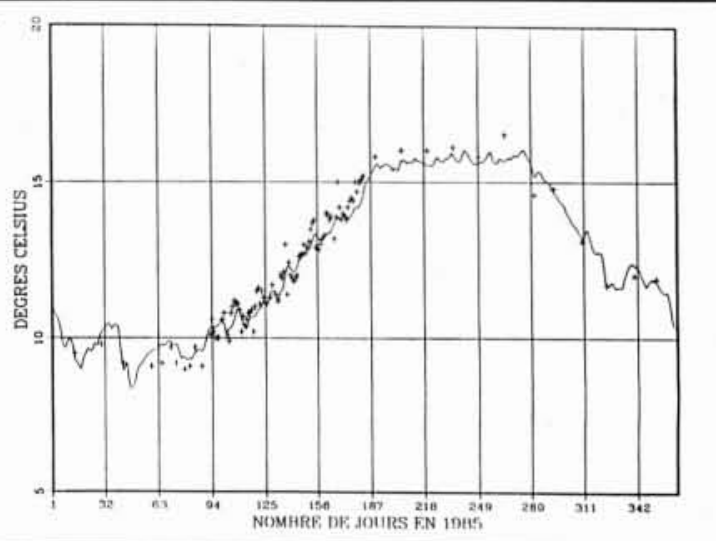

9.

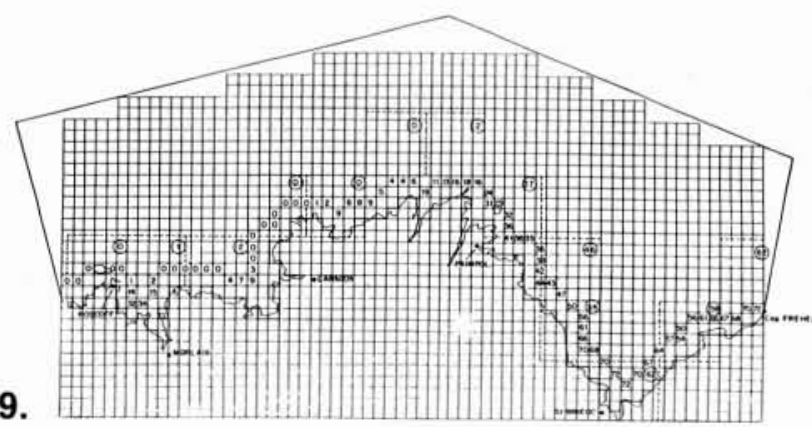



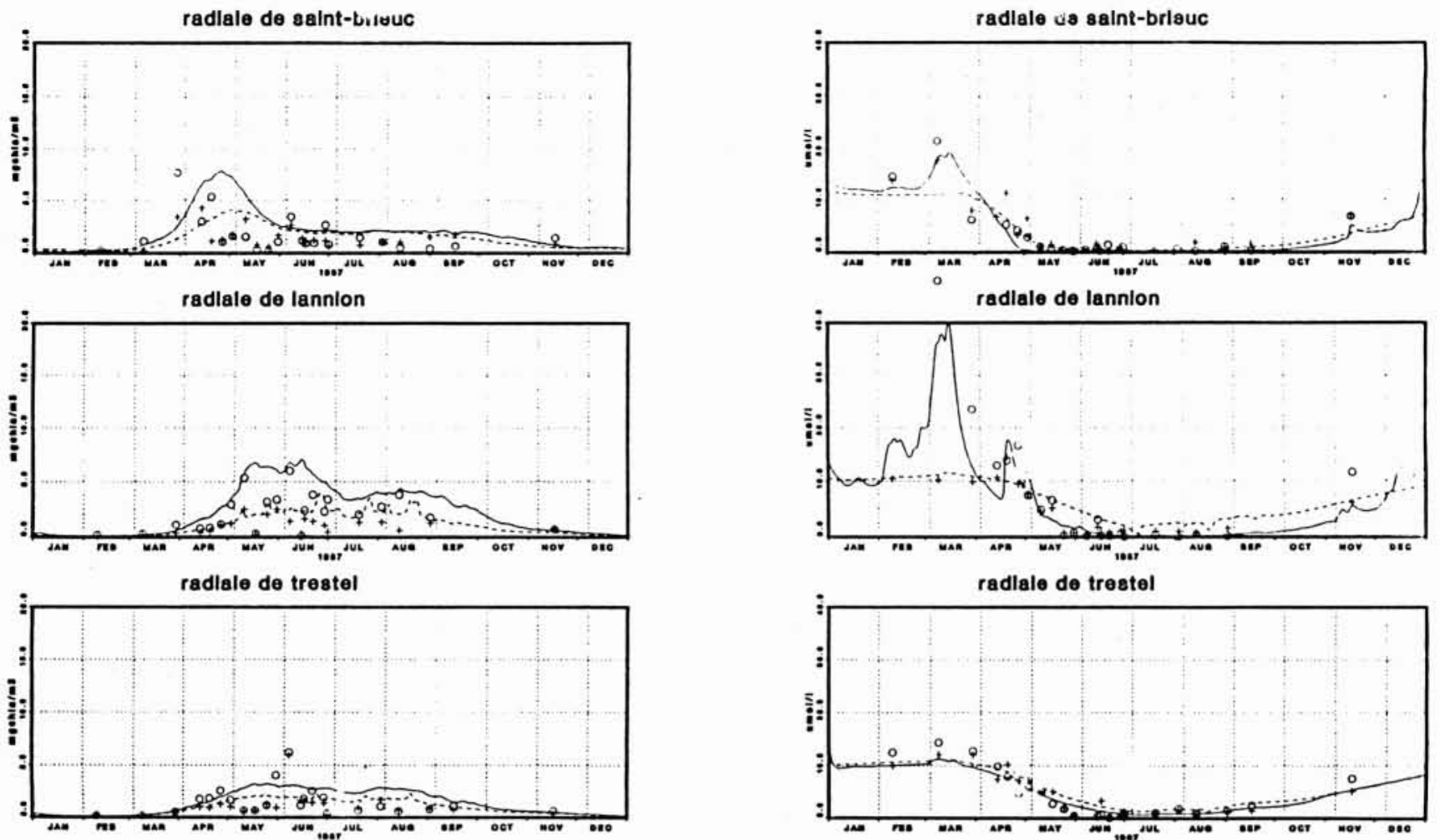

10. Chlorophylle a $\left(\mathrm{mgChla} / \mathrm{m}^{3}\right)$. Comparaison de l'évolution journalière simulée et des points de mesures aux extrémités côté large de chaque radiale.

11. Nitrates ( $\mu \mathrm{mol} / \mathrm{l}-1)$. Comparaison de l'évolution journalière simulée et des points de mesures aux extrémités côté large de chaque radiale.
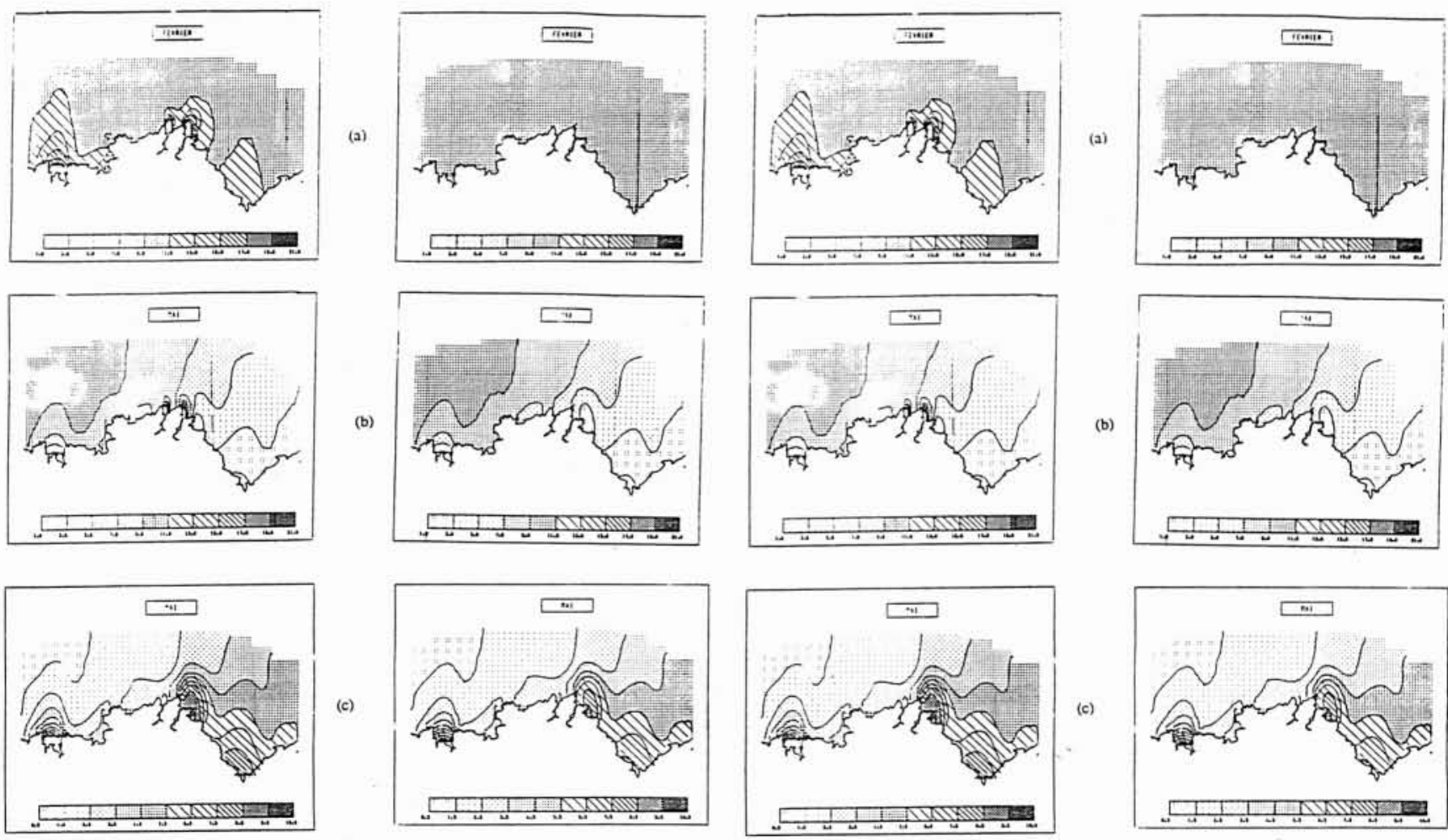

12. Modèle côtier complet. Concentration en (a) azote nitrique en février en surface ( $\mu$ atgN/l); (b) azote nitrique en mai en surface $(\mu \mathrm{atgN} / 1)$; (c) chlorophylle $a$ en mai en surface $\left(\mathrm{mg} \mathrm{CHla} / \mathrm{m}^{3}\right)$.
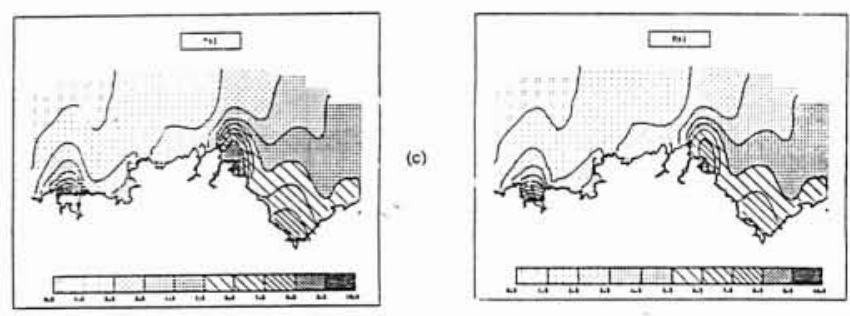

14. Modèle côtier complet, avec concentration initiale de la végétation algale benthique divisée par deux. Concentration en (a) azote nitrique en février en surface ( $\mu$ atgN/l); (b) azote nitrique en mai en surface ( $\mu \mathrm{atgN} / \mathrm{l})$; (c) chlorophylle $a$ en mai en surface $\left(\mathrm{mg} \mathrm{Chla} / \mathrm{m}^{3}\right)$.

13. Modèle côtier sans rejets. Concentration en (a) azote nitrique en février en surface ( $\mu \operatorname{atgN} / 1)$, (b) azote nitrique en mai en surface ( $\mu \operatorname{tg} \mathrm{N} / 1)$; (c) chlorophylle $a$ en mai en surface (mg Chla $/ \mathrm{m}^{3}$ ).

15. Modèle côtier sans rejet, avec concentration initiale de la végétation algale benthique divisée par deux. Concentration en (a) azote nitrique en février en surface ( $\mu$ atgN/l); (b) azote nitrique en mai en surface ( $\mu$ atgN/l); (c) chlorophylle $a$ en mai en surface $\left(\mathrm{mg} \mathrm{CHla} / \mathrm{m}^{3}\right)$. 

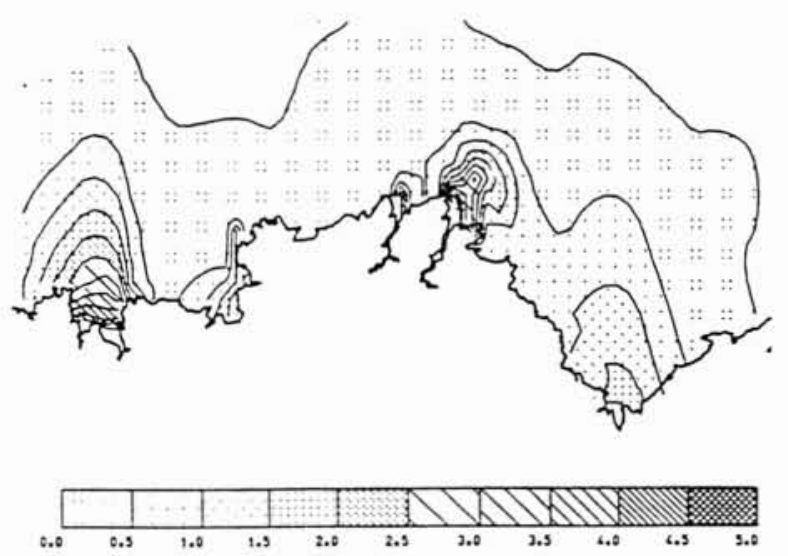

16. Différence entre les taux de production annuelle en phytoplancton $\left(\mathrm{gC} / \mathrm{m}^{2}\right)$ entre : - le modèle côtier complet; - le modèle côtier sans les rejets.
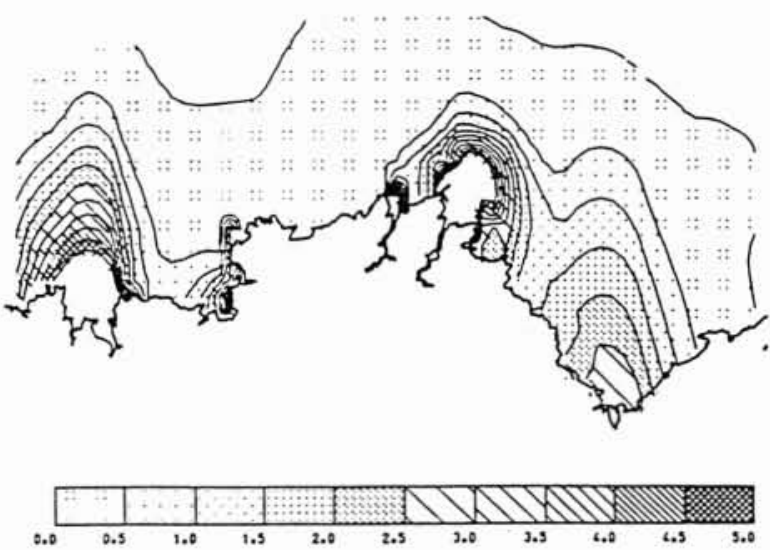

17. Différence entre les taux de production annuelle en phytoplancton $\left(\mathrm{gC} / \mathrm{m}^{2}\right)$ entre : - le modèle côtier complet ; - le modèle côtier sans les rejets. La concentration initiale de la végétation algale benthique étant réduite de $50 \%$ dans les deux cas. d'azote nitrique et de phytoplancton, variables qui sont extrêmement sensibles à la valeur de ces paramètres.

On présente sur les figures 10 et 11 la comparaison entre les courbes simulées par le modèle côtier et les points de mesure pour les variables chlorophylle $a$, indicateur de la biomasse phytoplanctonique et la concentration en nitrates.

L'importance de la végétation algale fixée sur les calculs du phytoplancton et de l'azote nitrique est clairement illustrée sur les figures 12 et 14 , où on y compare les résultats obtenus d'une part à l'aide de la version définitive du modèle côtier, et d'autre part à l'aide d'une simulation dans laquelle on suppose une diminution de $50 \%$ des concentrations initiales ( $1^{\mathrm{er}}$ janvier $)$ de la végétation fixée : on observe pour des fonds allant de 0 à 12 mètres, une augmentation de près de $55 \%$ de la production phytoplanctonique annuelle, qui passe de 46 à $70 \mathrm{gC} / \mathrm{m}^{2}$. On notera que dans le même temps, la proportion de la production phytoplanctonique dans la production primaire totale s'accroît de 22 à $46 \%$.

La végétation algale fixée, qui consomme l'azote plus précocement que le phytoplancton, exerce donc une rétention importante des ressources en sels nutritifs de la colonne d'eau et constitue un élément vital de l'écosystème côtier.

\section{Effet potentiel des apports d'azote d'origine fluviale sur le phytoplancton marin entre Roscoff et Saint- Brieuc}

Afin de tester l'impact des apports azotés d'origine fluviale sur l'évolution de la biomasse phytoplanctonique, nous avons utilisé à nouveau le modèle en supposant un arrêt total de tous ces rejets. Les résultats obtenus sont présentés sur l'ensemble du domaine, sous forme d'isovaleurs de surface en concentration de chlorophylle $a$ (mois de mai, période de plus forte production phytoplanctonique) et de nitrates (mois de février et mai) (fig. 13) et sont comparés à ceux issus du modèle côtier complet (fig. 12). Il apparaît que :

- en période hivernale, l'effet des apports azotés des rejets sur les concentrations en nitrates de la masse d'eau est spectaculaire, ce qui permet d'expliquer les fortes valeurs mesurées pour les points les plus côtiers au cours de la campagne SMART. Les eaux marines sont caractérisées par un gradient côte-large très marqué, les concentrations en nitrates les plus élevées étant atteintes en baie de Morlaix, à Lézardrieux et en baie de Saint-Brieuc $(>20$ $\mu a \operatorname{tgN} / 1)$. A cette époque de l'année, les poussées phyplanctoniques n'ont pas encore démarré, les conditions en température et en lumière étant trop sévères ;

- en fin de printemps, l'effet des apports côtiers est peu visible, que ce soit sur les teneurs en phytoplancton ou en azote nitrique. Le surplus d'éléments nutritits, apporté par les rivières, n'a donc apparemment pas d'impact sur l'accroissement des poussées phytoplanctoniques.

Cependant, si l'on raisonne, pour le phytoplancton, non plus en terme de concentration mais en production annuelle $\left(\mathrm{gC} / \mathrm{m}^{2}\right)$, les conclusions sont différentes. La figure 16 présente les écarts de production phytoplanctonique obtenus en simulant l'existence ou non des rejets d'azote : on constate un impact des rivières dans une bande côtière d'environ 10 kilomètres, se limitant toutefois au proche abord des embouchures des rivières. On observe pour des fonds inférieurs à 12 mètres, un excédent moyen de production phytoplanctonique entre Roscoff et SaintBrieuc de l'ordre de $4 \%$ par an, qui atteint plus de $10 \%$ dans les eaux marines proches de la zone de rejet des rivières Gouet, Leff et Trieux et de celles se jetant en baie de Morlaix.

L'impact des rejets azotés des rivières sur le phytoplancton est néanmoins pondéré par la présence de la végétation fixée benthique, dont on a mis en évidence précédemment l'effet de rétention vis-à-vis des réserves nutritives de la masse d'eau. $\mathrm{Si}$ on reprend le scénario - considéré au chapitre 3 - d'une division par deux de la concentration initiale de la végétation fixée, un arrêt de tous les rejets se traduit dans ce cas par un excédent moyen de production phytoplanctonique d'environ $7 \%$ sur la bande $0-12$ mètres avec des maximas voisins de $20 \%$ à l'approche de la zone de rejet des rivières. Il apparaît donc que l'impact des rejets azotés sur le phytoplancton est limité par la présence des algues benthiques. 


\section{Conclusion}

Le modèle numérique utilisé montre que sur le littoral Nord-Breton, l'impact des rejets azotés d'origine fluviale sur le phytoplancton marin est détectable. Il reste limité à une bande côtière dont la largeur n'excède pas 10 kilomètres, l'augmentation de la production annuelle phytoplanctonique dans la situation hydrologique de l'année 1987 étant de l'ordre de $4 \%$ pour des fonds inférieurs à 12 mètres. L'effet potentiel de ces apports sur la biomasse phytoplanctonique reste cependant limité dans les eaux bretonnes, du fait de l'abondance du couvert végétal d'origine benthique qui représente, dans le modèle, $78 \%$ de la production primaire pour des fonds inférieurs à 12 mètres. Ce compartiment benthique constitue, pour le phytoplancton, un compétiteur très sévère vis-à-vis de la consommation en nutriments, puisqu'il "pompe", avant le démarrage des poussées phytoplanctoniques printanières, une grande partie des rejets, afin de constituer ses propres réserves intracellulaires en azote.

Toute étude d'impact de rejets de polluants devrait donc, dans cette région, s'intéresser avant tout au compartiment benthique qui, par ses fortes capacités à stocker les nutriments même à basse température et à faible luminosité, " filtre" l'effet potentiel que peuvent exercer ces rejets sur la biomasse phytoplanctonique; c'est la voie adoptée récemment par MENESGUEN et SALOMON (1988) sur les problèmes d'Ulves en baie de Saint-Brieuc.

Ce modèle, qui reste perfectible - en particulier, il ne prend pas en compte, les flux d'azote à l'interface eausédiment, dont le rôle sur les réserves nutritives de la colonne d'eau sont encore aujourd'hui très contreversés (BOUCHER et Boucher-RodonI, 1985, LASSERre, 1987) constitue un outil de départ intéressant pour examiner à l'échelle côtière l'impact de rejets de toutes sortes, accidentels ou non, sur le début de la chaîne trophique.

\section{Références}

Assolre J. (1985). - Bilan en azote et en phosphore de la péninsule bretonne. DEA d'agronomie, Rennes.

Agoumı A. (1985), - Modélisation de l'écosystème pélagique en Manche ; étude de l'influence des phénomènes physiques sur le système planctonique. Thèse de doctorat d'état, université Pierre et Marie Curie, Paris $6^{\mathrm{e}}$.

Agoumi A., Gosse P. and Kalanskı M. (1985). - Numerical modelling of the influence of the vertical thermal structure on phytoplanktonic growth in the english channel. Proceedings of the nineteenth european marine biology symposium. Plymouth, Devon, U.K. 16-21 september 1984, Ed. P.E. Gibbs, p. 23-38.

BOUCHER G., Boucher-Rodoni R. (1985). - Fluctuations des nutriments au cours de la marée sur les parcs ostréicoles de la rivière penzé (Nord-Finistère). Hydrobi., 123, 251261.

Chasse C. (1983). - Le potentiel de production des végétaux marins des côtes de France. Biomasse actualité, $\mathrm{n}^{\circ}$ spécial, $n^{\circ} 3$, p. $8-11$.

Chasse C., Kerambrun L., Le Gal Y., Treguer P. (1985). Biomasse algale de Bretagne, potentiel énergétique. Groupe d'étude des énergies nouvelles, contrat avec l'établissement public régional de Bretagne.

Drew E.A., Ireland J.F., MUir C., Roberton W.H. and RobinSON J.D. (1982). - Photosynthesis, respiration and other factors influencing the growth of Laminaria ochroleuca below 50 metres in the straits of Messina. Mar. Ecol., 3 : 335-355.

EPPLEY R.W. (1972). - Temperature and phytoplancton growth in the sea. Fish Bull, 70, 1063-1085.

Gosse R.W. (1982). - Etude de la qualité des eaux de Doubs entre Longres et Dole, de mai à octobre 1981. Equations et étalonnage du modèle. Rapport EDF/DER/HE.31/82 $n^{\circ} 55$.

Gosse P., Riou J., VideAu C., à paraître en 1990. - Etude de l'écosystème planctonique en Manche sur la bande côtière Roscoff-Saint-Brieuc : résultats de la campagne de mesures $S M A R T$. Rapport EDF/DER/HE.31/90 n 1 .
Gosse P., Riou J. (1989). - Statistiques de température de la Manche le long des côtes Nord-Bretagne et OuestNormandie: années 1962 à 1987. Rapport EDF/ DER/HA/31/89 n 8 .

I.S.T.P.M. (1976). - Température et salinité de l'eau de mer au large de Roscoff au cours des années 1959 à 1970. Trav. Stat. Biol. Roscoff. 23, 31-49.

KerAmbrun L. (1984). - Contribution à l'étude de la fertilité des fonds rocheux cótiers de Bretagne. Thèse de $3^{\mathrm{e}}$ cycle, université de Bretagne occidentale, $150 \mathrm{pp}$.

KREMER J.N., Nixon S.W. (1978). - A coastal marine ecosystem, simulation and analysis. Springer-Verlag, Berlin, $217 \mathrm{pp}$.

LASSERRE P. (1987). - Echanges des sels minéraux dans les interfaces marines eau-sédiment, soumises à des turbulences provoquées ( effets tempête/coups de vent "). Rapport d'étude, contrat EDF n² 2 G 3661.

Menesguen A. and SAlomon J.C. (1988). - Eutrophication modelling as a tool for fighting against Ulva coastal mass blooms. Communication pour l'International Conference on computer modelling in ocean engineers. Venise 19-23 septembre 1988.

Regimbaud F. (1987). - Application de deux codes bidimensionnels en élèments finis à l'étude de l'impact thermique de centrales nucléaires. Projet de fin d'étude, Ecole Nationale des Ponts et Chaussées.

RILEY G.A. (1975). - Transparency-chlorophyll relations. Limnol. Oceanogr., 20, 150-152.

RıOU J., a paraitre en 1989. - Modélisation de l'écosystème planctonique sur le littoral nord-breton. Thèse de $3^{\mathrm{c}}$ cycle, Institut National Polytechnique de Toulouse.

SCIANDRIA (1982). - Etude d'un écosystème marin arfificiel. Thèse de docteur $3^{\mathrm{e}}$ cycle, Paris $6^{\mathrm{e}}$.

Sournia A., Birrien J.L., Douville J.L., Klein B. and Violler M. (1987). - A daily study of the Diatom Spring Bloom et Roscoff (France) in 1985. I. The Spring Bloom Within the Annual Cycle. Estuarine, Coastal and Shelf Science (1987) 25, 355-367. 